\title{
Hybrid Reference Function for Stable Stepwise Inertial Control of a Doubly-Fed Induction Generator
}

\author{
Dejian Yang*, Jinsik Lee*, Kyeon Hur** and Yong Cheol Kang ${ }^{\dagger}$
}

\begin{abstract}
Upon detecting a frequency event in a power system, the stepwise inertial control (SIC) of a wind turbine generator (WTG) instantly increases the power output for a preset period so as to arrest the frequency drop. Afterwards, SIC rapidly reduces the WTG output to avert over-deceleration (OD). However, such a rapid output reduction may act as a power deficit in the power system, and thereby cause a second frequency dip. In this paper, a hybrid reference function for the stable SIC of a doublyfed induction generator is proposed to prevent OD while improving the frequency nadir (FN). To achieve this objective, a reference function is separately defined prior to and after the FN. In order to improve the FN when an event is detected, the reference is instantly increased by a constant and then maintained until the FN. This constant is determined by considering the power margin and available kinetic energy. To prevent OD, the reference decays with the rotor speed after the FN. The performance of the proposed scheme was validated under various wind speed conditions and wind power penetration levels using an EMTP-RV simulator. The results clearly demonstrate that the scheme successfully prevents OD while improving the FN at different wind conditions and wind power penetration levels. Furthermore, the scheme is adaptive to the size of a frequency event.
\end{abstract}

Keywords: Doubly-fed induction generator, Minimum rotor speed, Over-deceleration, Stepwise inertial control, Second frequency dip.

\section{Introduction}

The system frequency, which indicates a balance between power generation and consumption in a power system, should be kept within an allowable range at all times. If a mismatch between generation and consumption appears due to a large generator trip or a sudden load variation, synchronous generators (SGs) naturally release their kinetic energy (KE) stored in the rotating masses as an inertial response. The SGs then participate in primary control to arrest the system frequency drop [1].

Variable-speed wind turbine generators (WTGs), including doubly-fed induction generators (DFIGs) and fully rated converter-based WTGs, have been widely used because they perform maximum power point tracking (MPPT) control in order to capture maximum energy from wind. However, MPPT decouples the WTG frequency from the system frequency, thereby reducing system inertia. Consequently, frequency stability in the power system is jeopardized, particularly at high wind power penetration levels [2]. WTGs are thus forced or recommended to participate in frequency control so as to facilitate frequency

$\dagger$ Corresponding Author: Dept. of Electrical Engineering, WeGAT Research Center, and Smart Grid Research Center, Chonbuk National University, Korea. (yckang@jbnu.ac.kr)

* Dept. of Electrical Engineering and WeGAT Research Center, Chonbuk National University, Korea. (dejian@jbnu.ac.kr, jinsiklee @jbnu.ac.kr)

* School of Electrical and Electronic Engineering, Yonsei University, Seoul, Korea. (khur@yonsei.ac.kr)

Received: June 30, 2015; Accepted: October 11, 2015 stability in a power system [3].

In a number of studies on inertial control in WTGs, it has been reported that the output power can be increased by releasing the KE stored in the rotating masses of WTGs. Inertial control can be divided into two groups: frequency based inertial control (FBIC) [4-9] and stepwise inertial control (SIC) [10-15]. Based on the measured frequency, FBIC employs one or two auxiliary loops: a rate of change of frequency (ROCOF) loop [4], a frequency deviation loop [5], ROCOF and frequency deviation loops [6], and maximum ROCOF and frequency deviation loops [7]. While FBIC contributes to an increase in the frequency nadir (FN), it shows a relatively slow response, because the additional power from the auxiliary loops relies on frequency variations. On the other hand, to improve the performance of inertial control, the gain of frequency deviation loop was regulated depending on the rotor speed of WTGs [8] and the ROCOF [9].

To provide a faster response when a disturbance is detected, SIC switches the power reference for MPPT control to the reference for SIC. The reference is then instantly increased and maintained for a predefined time. Because the output is instantly increased at the initial stage of an event without using the measured frequency, SIC improves the FN more than FBIC. However, SIC schemes should decrease the output to prevent over-deceleration (OD) $[10-13]$. Such a decrease may act as a power deficit in a power system, thereby causing a second frequency dip (SFD). To reduce this drawback, ramp reduction has been 
suggested as an alternative to instant reduction [14, 15]. While these schemes partly lessen the degree of an SFD, they are unable to completely prevent it. Furthermore, conventional SIC schemes may cause OD during inertial control under low wind conditions.

In this work, a hybrid reference function for the stable SIC of a DFIG is proposed to prevent OD while improving the FN. To achieve this objective, the proposed reference function is separately defined prior to and after the FN. In order to improve the FN when an event is detected, the reference is increased by a constant and maintained until the FN. This constant is determined by considering the power margin and available KE. To prevent OD, the reference decreases with the rotor speed after the FN. The performance of the proposed scheme was validated under various wind conditions and wind power penetration levels using an EMTP-RV simulator.

\section{Conventional SIC Schemes of a DFIG}

Fig. 1 illustrates the operational features of two conventional SIC schemes proposed in [10] and [14]. For convenience, the SICs [10] and [14] are denoted as SIC \#1 and SIC \#2, respectively. Upon detecting an event, SIC \#1 and SIC \#2 switch the reference for MPPT control, $P_{M P P T}$, to the reference for SIC, $P_{S I C}$, as shown in Fig. 1(a). The $P_{S I C}$ in SIC \#1 and SIC \#2 consists of two periods: an overproduction period, $T_{O P}$, and an underproduction period,

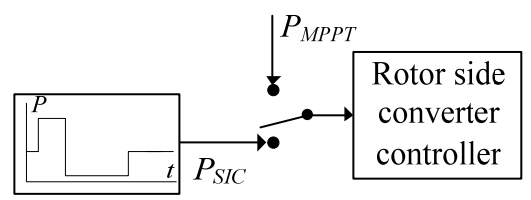

(a) Conventional SIC schemes

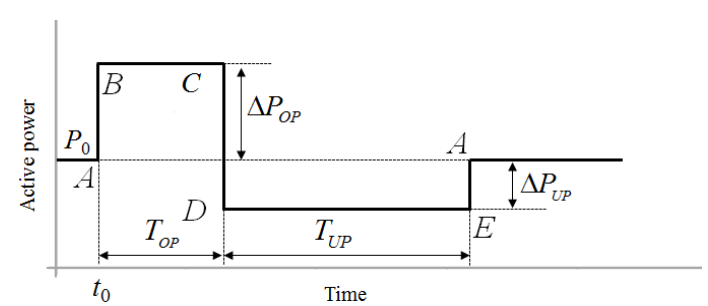

(b) Reference function of SIC \#1 [10]

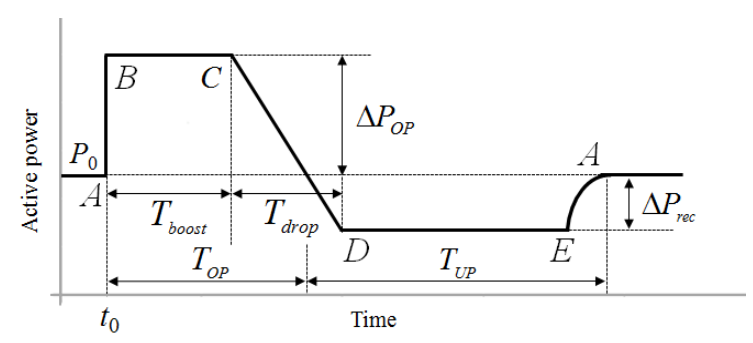

(c) Reference function of SIC \#2 [14]

Fig. 1. Operational characteristics of SIC \#1 and SIC \#2
$T_{U P}$ (see Figs. 1(b) and 1(c)). In both schemes, a constant $\Delta P_{O P}$ is added to $P_{0}$, which represents the power reference prior to an event. This means that $P_{S I C}$ instantly increases from point $A$ to point $B$ and is maintained from point $B$ to point $C$.

To prevent OD, SIC \#1 rapidly reduces the output at point $C$, which means that $P_{S I C}$ moves from point $C$ to point $D$ in Fig. 1(b). Such a large reduction rate may cause an SFD. In order to minimize this problem, SIC \#2 reduces its output in a ramp-like rather than step-like manner (see Fig. 1(c)). To ensure a slow reduction rate, a large $T_{\text {drop }}$ is desirable. However, an excessively large $T_{\text {drop }}$ extracts too much KE from a WTG, which could in turn cause OD. Conversely, an excessively small $T_{d r o p}$ results in a rapid output drop, thereby causing an SFD. In [14], $10 \mathrm{~s}$ is used for $T_{d r o p}$. Consequently, SIC \#2 partly lessens the degree of an SFD, but is unable to completely prevent it.

In SIC \#1, $\Delta P_{O P}$ and $\Delta P_{U P}$ are assigned values of 0.10 p.u. and 0.05 p.u., respectively, while $T_{O P}$ and $T_{U P}$ are respectively set to $10.0 \mathrm{~s}$ and $20.0 \mathrm{~s}$. Conversely, $\Delta P_{O P}$ is determined by the $\mathrm{KE}$ in SIC \#2 so as to improve the FN more than in SIC \#1, whereas $\Delta P_{\text {rec }}$ is set to 0.05 p.u. (as in SIC \#1). Unlike SIC \#1, $T_{U P}$ is not fixed and lasts until $P_{S I C}$ reaches $P_{M P P T}$.

\section{Proposed Hybrid Reference Function for SIC}

This proposed SIC scheme aims to (1) improve the FN more than in conventional SIC schemes and (2) prevent OD by converging the rotor speed into a stable operating region. To achieve these objectives, a hybrid reference function separately defined prior to and after the $\mathrm{FN}$ is proposed. Fig. 2 illustrates the operational features of the proposed scheme.

To achieve the first goal, $P_{S I C}$ is defined in the time domain and maintained until the FN. Here, $P_{S I C}$ may be expressed as

$$
P_{S I C}=P_{0}+\Delta P
$$

where $P_{0}$ is the power reference prior to a frequency event and $\Delta P$ is a constant.

To raise the $\mathrm{FN}, \Delta P$ in (1) is defined as

$$
\Delta P=\left(P_{\text {Tlimit }}-P_{0}\right) \times \frac{\omega_{0}^{2}-\omega_{\min }^{2}}{\omega_{\max }^{2}-\omega_{\min }^{2}}
$$

where $P_{\text {Tlimit }}, \omega_{0}, \omega_{\min }$, and $\omega_{\max }$ are the torque limit referred to power, the rotor speed prior to an event, and the minimum and maximum rotor speeds, respectively.

The first term on the right hand side in (2) is the power margin, defined as the difference between $P_{\text {Tlimit }}$ and $P_{0}$. Both $P_{\text {Tlimit }}$ and $P_{0}$ depend on the wind and rotor speeds. The second term, which is a weighting factor of the power 


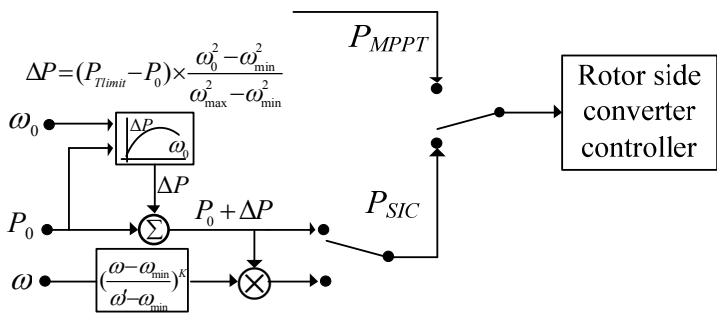

(a) Control scheme

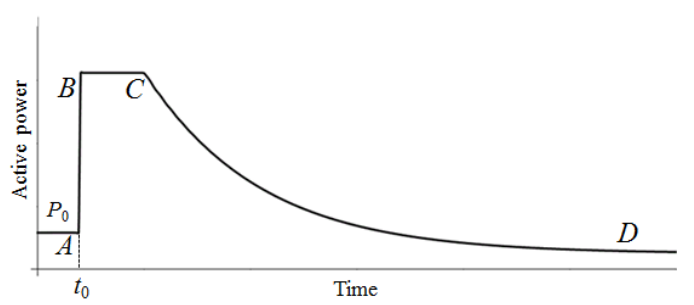

(b) Proposed reference function

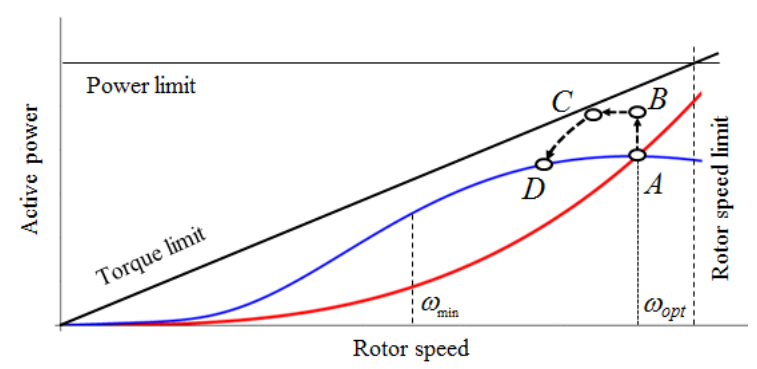

(c) Power-speed locus $(K=0.5)$

Fig. 2. Operational characteristics of the proposed scheme

margin, is defined as the ratio of the releasable $\mathrm{KE}$ of a WTG to the maximum releasable KE. Note that the weighting factor is set to 1 at $\omega_{\max }$ and 0 at $\omega_{\min }$. This means that, at $\omega_{\max }, \Delta P$ is set to the power margin in order to improve the $\mathrm{FN}$, whereas at $\omega_{\min }, \Delta P$ is set to 0 to avoid OD. Fig. 3 illustrates the relationship between $\Delta P$ and $\omega_{0}$ at various rotor speeds for the proposed scheme. In the proposed scheme, $\Delta P$ has the maximum value at $\omega_{0}$ of 1.14 p.u.

As shown in Fig. 2(b), $P_{S I C}$ instantly increases from point $A$ to point $B$, and is maintained from point $B$ to point $C$. This locus from point $A$ to point $C$ shows a pattern similar to those of SIC \#1 and SIC \#2. However, to achieve the second objective, $P_{S I C}$ is defined after the $\mathrm{FN}$ as

$$
P_{S I C}=\left(P_{0}+\Delta P\right) \times\left(\frac{\omega-\omega_{\min }}{\omega^{\prime}-\omega_{\min }}\right)^{K}
$$

where $\omega, \omega$, and $K$ are the rotor speed, rotor speed at the $\mathrm{FN}$, and a quotient, respectively.

The reference in the form of (3) ensures stable operation of a DFIG during inertial control because it prevents OD. The reason for this is as follows. As shown in Fig. 2(c), $P_{S I C}$ meets the mechanical input, $P_{m}$, at point $D$ under various wind conditions. Point $D$ is also located within a

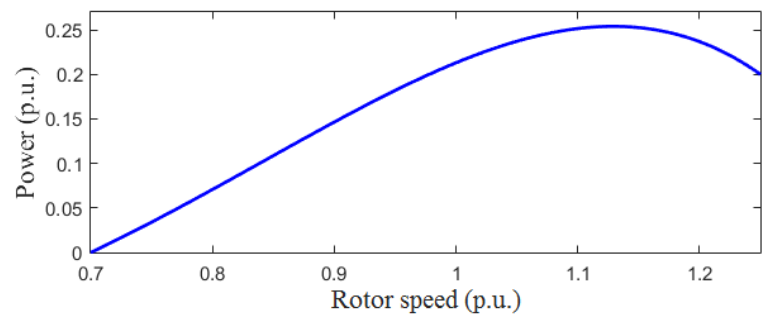

Fig. 3. $\Delta P$ versus $\omega_{0}$ for the proposed scheme

stable operating range, i.e., between $\omega^{\prime}$ and $\omega_{\min }$. Thus, the rotor speed stays within a stable operating region.

From (3), it can also be seen that $P_{S I C}$ decreases as $\omega$ is reduced. This means that the output of the DFIG gradually decreases. As shown in Fig. 2(b), $P_{S I C}$ exhibits a smooth decrease from point $C$ to point $D$, thereby helping to prevent an SFD.

To explain this scenario, differentiation of (3) gives

$$
\frac{d P_{S I C}}{d t}=\left(P_{0}+\Delta P\right) \times K\left(\frac{\omega-\omega_{\min }}{\omega^{\prime}-\omega_{\min }}\right)^{K-1} \frac{d \omega}{d t}
$$

The reduction rate of $P_{S I C}, d P_{S I C} / d t$, remains at a small value except in the initial stage. This serves to facilitate SFD prevention.

To improve the FN upon detecting an event, the proposed scheme instantly increases the output until the FN. After the FN, the output is reduced with the rotor speed to prevent OD; this ensures stable operation of a DFIG. Furthermore, an SFD can be avoided due to the small output reduction rate.

\section{Model System}

Fig. 4 shows the model system used in this study to investigate the performance of the proposed scheme; it includes $900 \mathrm{MW}$ SGs, $550 \mathrm{MW}$ motor and static loads, and a $100 \mathrm{MW}$ DFIG-based wind power plant (WPP). The model system is simulated using an EMTP-RV simulator.

\subsection{SGs}

Six SGs are included in the model: two 200 MVA SGs,

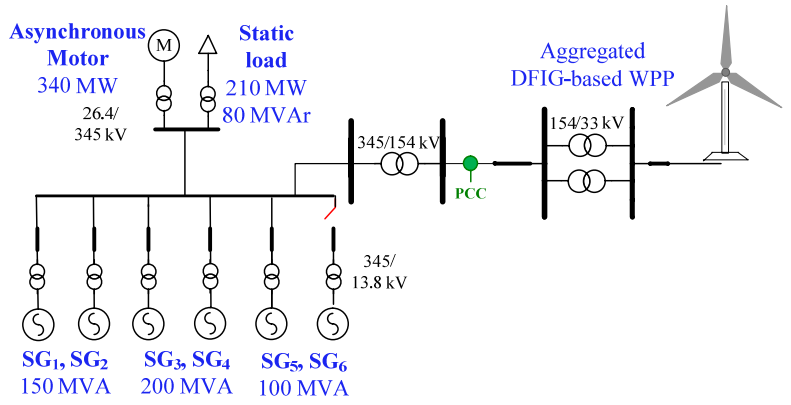

Fig. 4. Model system employed in this work 
two 150 MVA SGs, and two 100 MVA SGs. All SGs are assumed to be steam turbine governors to benchmark the Korean power system, which has a low ramping capability. In addition, IEEEG1 is used to model the steam turbine governors [16]; the droop gain of the SGs was set to 5\%, a typical value for the governor setting.

\subsection{DFIG Model}

Fig. 5 illustrates the typical configuration of a DFIG, which consists of a wind turbine model, two-mass shaft model, and DFIG controllers [3].

The mechanical input power extracted from the wind, $P_{m}$, can be represented by

$$
P_{m}=\frac{1}{2} \rho \pi R^{2} v^{3} c_{P}(\lambda, \beta)
$$

where $\rho, R, v, c_{P}, \lambda$, and $\beta$ are the air density, blade length, wind speed, power coefficient, tip speed ratio, and pitch angle, respectively. Furthermore, $c_{P}$ may be expressed as

$$
c_{P}(\lambda, \beta)=0.645\left\{0.00912 \lambda+\frac{-5-0.4(2.5+\beta)+116 \lambda_{i}}{e^{21 \lambda_{i}}}\right\}
$$

where

$$
\lambda_{i}=\frac{1}{\lambda+0.08(2.5+\beta)}-\frac{0.035}{1+(2.5+\beta)^{3}}
$$

Because (6) is used in this work, the maximum $c_{p}$ and optimal $\lambda$ become 0.5 and 9.95 , respectively [17].

A two-mass shaft model is represented by

$$
\left.\begin{array}{l}
2 H_{t} \frac{d \omega_{t}}{d t}=T_{t}-K_{s} \theta_{s}-D_{s}\left(\omega_{t}-\omega_{r}\right)-D_{t} \omega_{t} \\
2 H_{g} \frac{d \omega_{r}}{d t}=K_{s} \theta_{s}+D_{s}\left(\omega_{t}-\omega_{r}\right)-D_{g} \omega_{r}-T_{g} \\
\frac{d \theta_{s}}{d t}=\omega\left(\omega_{t}-\omega_{r}\right)
\end{array}\right\}
$$

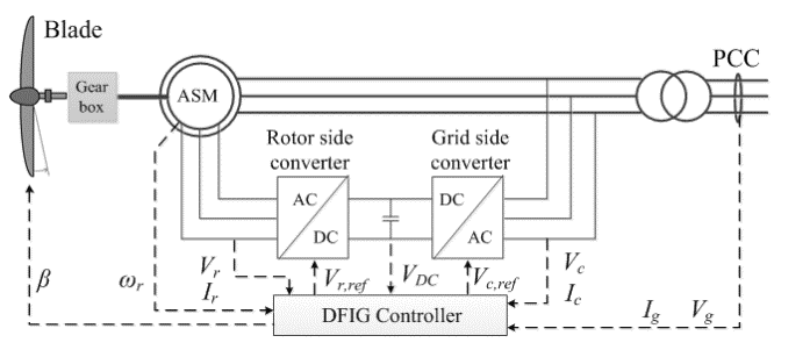

$V_{r}, I_{r}$ : voltage and current at the rotor circuit,

$V_{c}, I_{c}$ : voltage and current at the GSC

$V_{g}, I_{g}$ : voltage and current at the point of common coupling (PCC)

$V_{r, r e f}, V_{c . r e f}$ : voltage reference for the RSC and GSC

$\omega_{r}$ : rotor speed, $\beta$ : pitch angle, $V_{D C}$ : DC-link voltage

Fig. 5. Typical configuration of a DFIG

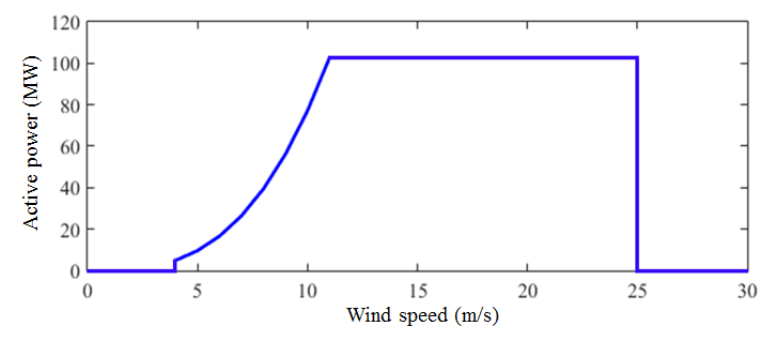

Fig. 6. Power curve of the WPP used in this study

where $H_{t}, H_{g}, \omega_{t}, \omega_{r}, T_{t}, T_{g}, D_{t}$, and $D_{g}$ are the inertia time constants, angular speeds, torques, and damping constants of a wind turbine and a generator mass, respectively; $K_{s}, D_{s}$, $\theta_{s}$, and $\omega$ are the shaft stiffness, damping constant, torsional twist, and base value of angular speed, respectively.

The DFIG controller determines the reference signals for a rotor-side convertor (RSC) and grid-side converter (GSC). The RSC controls the active and reactive power injected into a power system, whereas the GSC maintains the DClink and controls terminal voltages.

\subsection{DFIG-based WPP}

As shown in Fig. 6, the cut-in, rated, and cut-out wind speeds are $4 \mathrm{~m} / \mathrm{s}, 11 \mathrm{~m} / \mathrm{s}$, and $25 \mathrm{~m} / \mathrm{s}$, respectively. The stable operating range of the DFIG is from 0.7 p.u. to 1.25 p.u. To obtain realistic results, both power and torque limitations are considered here. The power limit was set to 1.2 p.u., while the maximum torque limit was set to 1.17 p.u.

\section{Case Studies}

The performance of the proposed scheme under various wind conditions and wind power penetration levels will now be discussed. Here, wind speeds of $11 \mathrm{~m} / \mathrm{s}$ and $7 \mathrm{~m} / \mathrm{s}$ were utilized, while wind power penetration levels of $18.2 \%$ and $32.7 \%$ were employed.

As a frequency event, $\mathrm{SG}_{6}$, which is generating $70 \mathrm{MW}$ before the event, is tripped out at $40.0 \mathrm{~s}$. In SIC \#1, $T_{O P}$ and $T_{U P}$ are assigned values of $10.0 \mathrm{~s}$ and $20.0 \mathrm{~s}$, respectively, while $\Delta P_{O P}$ and $\Delta P_{U P}$ are respectively set to 0.10 p.u. and 0.05 p.u. In SIC \#2, $T_{O P}$ and $T_{\text {drop }}$ are assigned values of $20.0 \mathrm{~s}$ and $10.0 \mathrm{~s} ; \Delta P_{U P}$ is set to different values under various wind conditions, whereas a value of $0.05 \mathrm{p} . \mathrm{u}$ is used for $\Delta P_{r e c}$. To achieve fast frequency recovery, $K$ is set to 0.5 in the proposed scheme. Note that secondary frequency control of a power system is not considered in this study and thus, the system frequency does not completely recover to $60 \mathrm{~Hz}$ in the case studies. However, such an issue was not addressed, as it lies beyond the scope of this work.

Case 1: Wind Speed of $11 \mathrm{~m} / \mathrm{s}$ and Wind Power Penetration Level of $18.2 \%$ 


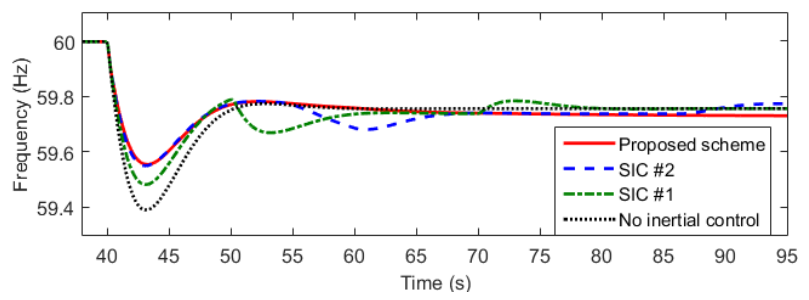

(a) System frequency

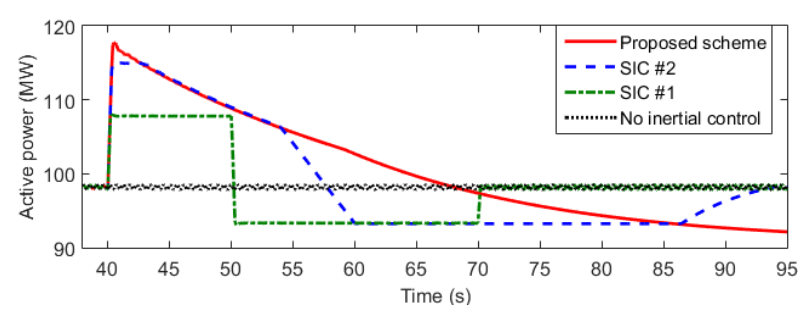

(b) Active power of the WPP

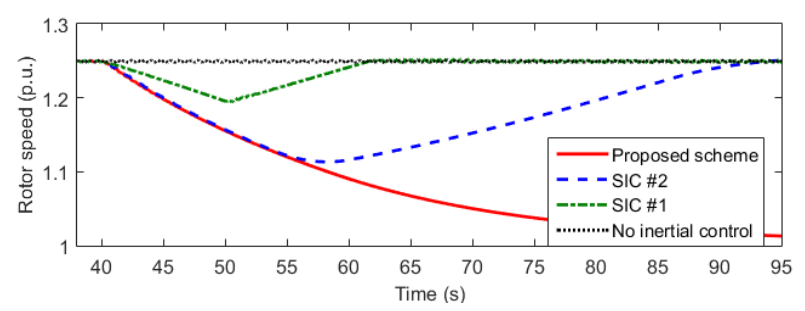

(c) Rotor speed

Fig. 7. Results for Case 1

Fig. 7 shows the results obtained for Case 1, in which the wind speed is the rated wind speed and thus, maximum $\mathrm{KE}$ is stored in the rotating masses of the DFIG. In the proposed scheme and SIC \#2, $\Delta P$ was set to 0.24 p.u. and 0.18 p.u., respectively. As shown in Fig. 7(a), the FNs for "no inertial control," SIC \#1, SIC \#2, and the proposed scheme are $59.39 \mathrm{~Hz}, 59.48 \mathrm{~Hz}, 59.54 \mathrm{~Hz}$, and $59.55 \mathrm{~Hz}$, respectively. This is because the proposed scheme releases a slightly larger output than SIC \#2 until the FN (at $43.1 \mathrm{~s}$ ), and a significantly larger output than SIC \#1 after an event (see Fig. 7(b)). Note that in this case, SIC \#1, SIC \#2, and the proposed scheme do not cause OD due to the large amount of KE.

The proposed scheme does not cause an SFD, as the rate of change in the output is smaller than that in SIC \#1 and SIC \#2. In SIC \#1, the output is maintained until $50.0 \mathrm{~s}$, at which point it instantly decreases. Consequently, a large SFD begins at $50.0 \mathrm{~s}$. In SIC \#2, the output decreases along with $P_{\text {Tlimit }}$ up to $54.2 \mathrm{~s}$, which means that the reference exceeds $P_{\text {Tlimit }}$. The output then starts decreasing in a ramplike manner. Thus, when compared to SIC \#1, a smaller and slower SFD starts in SIC \#2 at $53.8 \mathrm{~s}$.

In SIC \#1, the rotor speed decreases from $40.0 \mathrm{~s}$ to 50.0 $\mathrm{s}$, and then recovers from $50.0 \mathrm{~s}$ to $70.0 \mathrm{~s}$. In SIC \#2, the rotor speed decreases from $40.0 \mathrm{~s}$ to $57.0 \mathrm{~s}$, and then recovers after $57.0 \mathrm{~s}$. Conversely, the rotor speed for the proposed scheme decreases and eventually converges to

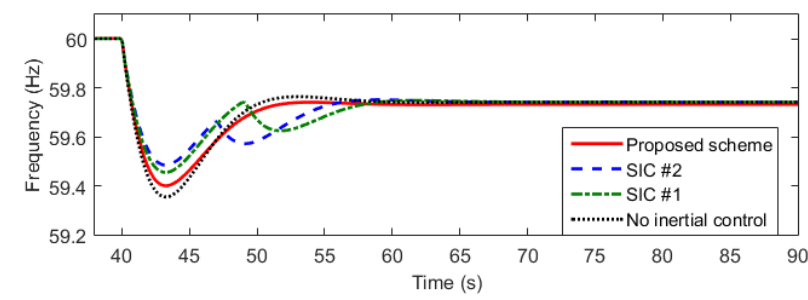

(a) System frequency

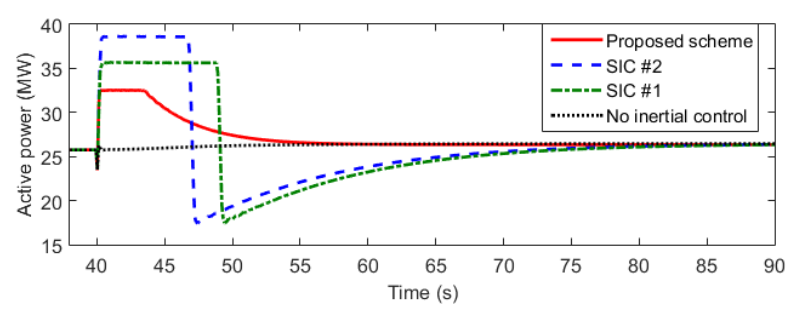

(b) Active power of the WPP

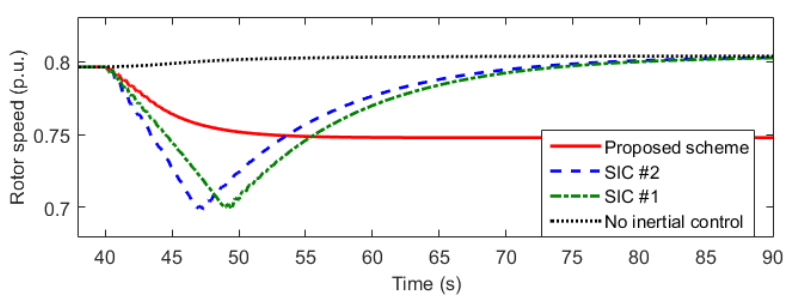

(c) Rotor speed

Fig. 8. Results for Case 2

1.01 p.u.

Case 2: Wind Speed of $7 \mathrm{~m} / \mathrm{s}$ and Wind Power Penetration Level of $18.2 \%$

Fig. 8 shows the results for Case 2, which is identical to Case 1 except for the smaller wind speed. Thus, $\Delta P$ for the proposed scheme and SIC \#2 are set to 0.07 p.u. and 0.13 p.u., respectively. Note that in the proposed scheme, $\Delta P$ is reduced to $29 \%$ of that obtained in Case 1, whereas in SIC $\# 2$, it is reduced to $72 \%$ of the $\Delta P$ in Case 1 .

In Case 2, SIC \#1 and SIC \#2 cause OD, while no OD is produced with the proposed scheme (see Fig. 8(c)). Consequently, SIC \#1 and SIC \#2 produce SFDs, but they are caused by OD rather than a significant output reduction rate, as in Case 1. As shown in Fig. 8(a), the FN of the proposed scheme is $59.40 \mathrm{~Hz}$, which is $0.03 \mathrm{~Hz}$ higher than that for "no inertial control", but lower than those of SIC $\# 1$ and SIC \#2. This is because SIC \#1 and SIC \#2 provide a larger $\Delta P$ than the proposed scheme (see Fig. $8(\mathrm{~b})$ ). Thus, OD occurs at $48.8 \mathrm{~s}$ for SIC \#1 and $46.7 \mathrm{~s}$ for SIC \#2. At those particular times, SIC is disabled, leading to a sizable decrease in the outputs of SIC \#1 and SIC \#2. Note that in this case, the second FNs in SIC \#1 and SIC \#2 are larger than the first FNs.

As in Case 1, the rotor speed in the proposed scheme decreases and eventually converges to 0.74 p.u., which is within the stable operating range. In contrast, the rotor speeds in SIC \#1 and SIC \#2 reach a minimum and then 


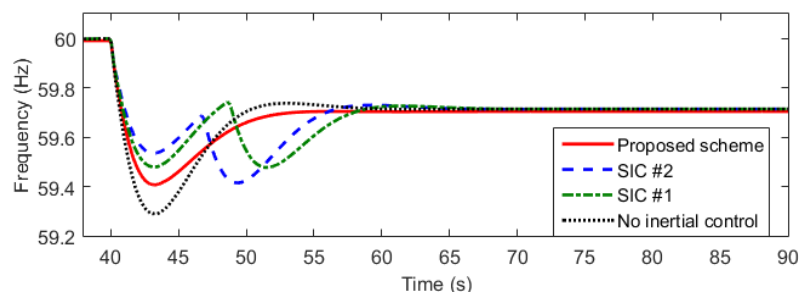

(a) System frequency

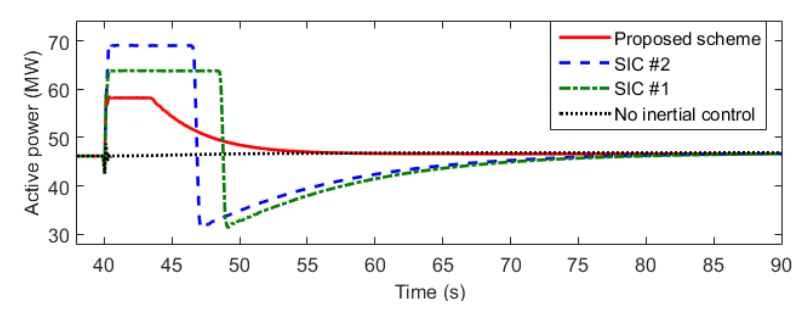

(b) Active power of the WPP

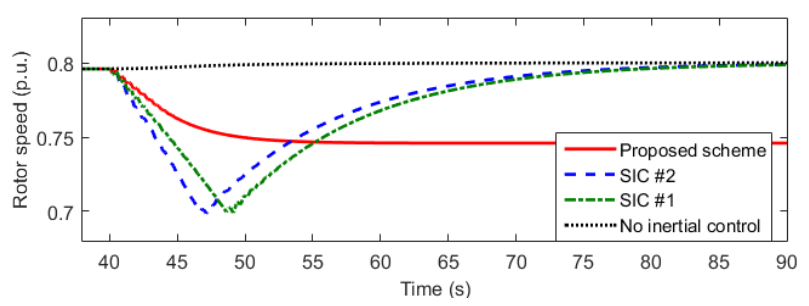

(c) Rotor speed

Fig. 9. Results for Case 3

increase because of the disabled SIC.

Case 3: Wind Speed of $7 \mathrm{~m} / \mathrm{s}$ and Wind Power Penetration Level of $32.7 \%$

Fig. 9 shows the results for Case 3, which is identical to Case 2 except for a higher wind power penetration level. In this case, the $\Delta P$ values for the proposed scheme and SIC \#2 are the same as those obtained for Case 2 due to the identical wind conditions.

As expected, the FN for "no inertial control" decreases to $59.29 \mathrm{~Hz}$ because of the reduced system inertia. The proposed scheme increases the FN by $0.13 \mathrm{~Hz}$ to $59.42 \mathrm{~Hz}$. However, as in Case 2, this value is smaller than the first FNs of SIC \#1 and SIC \#2 since $\Delta P$ in these SICs is larger than that in the proposed scheme.

Note that the second FNs in SIC \#1 and SIC \#2 are $59.47 \mathrm{~Hz}$ and $59.41 \mathrm{~Hz}$, respectively, which are lower than the first FNs. Furthermore, the second FN of SIC \#2 is lower than the FN of the proposed scheme. The reason for this is as follows. As in Case 2, the two conventional SICs release more power than in the proposed scheme at a low wind condition. Therefore, OD occurs at $48.6 \mathrm{~s}$ for SIC \#1 and $46.8 \mathrm{~s}$ for SIC \#2. In contrast, the proposed scheme does not cause OD. The significant output reduction rate in SIC \#1 and SIC \#2 causes an SFD, while no SFD is produced in the proposed scheme. The value of $\Delta P$ for SIC \#2 is larger than that for SIC \#1 and thus, OD occurs earlier than in SIC \#1.
As in Case 2, the rotor speed in the two conventional schemes reaches a minimum operating speed. However, the rotor speed in the proposed scheme decreases and eventually converges to 0.74 p.u., which is the same value reached in Case 2.

\section{Conclusions}

In this paper, a hybrid reference function for the stable SIC of a DFIG is proposed to prevent OD while improving the FN. To achieve this objective, the proposed reference is separately defined prior to and after the FN. In order to improve the $\mathrm{FN}$, the reference is instantly increased by additional power prior to the $\mathrm{FN}$, and then maintained until the FN. After the FN, the reference decreases with the rotor speed to prevent OD.

The obtained results demonstrate that the proposed scheme can prevent OD while improving the FN under various wind conditions and wind power penetration levels. In addition, the rotor speed of a DFIG converges within a stable operating region. The proposed reference also results in a small output reduction rate, thereby preventing an SFD.

The advantage of the proposed scheme is that it provides fast frequency support to a power system and stable operation of a DFIG under various wind conditions. Furthermore, the scheme is adaptive to both the size of an event and the level of inertia available in a power system.

\section{Acknowledgments}

This work was partly supported by the National Research Foundation of Korea (NRF) grant funded by the Korea government (MSIP) (No. 2010-0028509) and partly by research funds of Chonbuk National University in 2014.

\section{References}

[1] T. Ackermann, Wind Power in Power System, 2nd Edition, U.K.: John Wiley \& Sons, Ltd, 2012.

[2] S. E. Lee, D.-J. Won and I.-Y. Chung, "Operation scheme for a wind farm to mitigate output power variation," J. Elect. Eng. Technol, vol. 7, no. 6, pp. 869-875, Nov. 2012.

[3] O. Anaya-lara, N. Jenkins, J. Ekanayake, P. Cartwright, and M. Hughes, Wind Energy Generation Modeling and Control, U.K.: John Wiley \& Sons, Ltd, 2009.

[4] J. Ekanayake and N. Jenkins, "Comparison of the response of doubly fed and fixed-speed induction generator wind turbine to changes in network frequency," IEEE Trans. Energy Convers., vol. 19, no. 4, pp. 800-802, Dec. 2004.

[5] J. Morren, J. Pierik, and S. W. H. de Haan, "Inertial 
response of variable speed wind turbines," Electric Power Systems Research, vol. 76, no. 1, pp. 980-987, Dec. 2006.

[6] J. Morren, S. Haan, W. L. Kling, and J. A. Ferreira, "Wind turbines emulating inertia and supporting primary frequency control," IEEE Trans. Power Syst., vol. 21, no. 1, pp. 433-434, Feb. 2006.

[7] H. Lee, J. Kim, D. Hur, and Y. C. Kang, "Inertial control of a DFIG-based wind power plant using the maximum rate of change of frequency and the frequency deviation," J. Elect. Eng. Technol, vol. 10, no.2, pp. 496-503, Mar. 2015.

[8] J. Lee, J. Kim, Y.-H. Kim, Y.-H. Chun, S.-H. Lee, J.K. Seok, and Y. C. Kang, "Rotor speed-based droop of a wind generator in a wind power plant for the virtual inertial control," J. Elect. Eng. Technol, vol. 8, no. 5, pp. 742-749, Sep. 2013.

[9] M. Hwang, Y.-H. Chun, J.-W. Park, and Y.C. Kang, "Dynamic Droop-based Inertial Control of a Wind Power Plant," J. Elect. Eng. Technol, vol. 10, no. 3, pp. 1363-1369, May 2015.

[10] N. R. Ullah, T. Thiringer, and D. Karlsson, "Temporary primary frequency control support by variable speed wind turbines - Potential and applications," IEEE Trans. Power Syst., vol. 23, no. 2, pp. 601-612, May 2008.

[11] G. Tarnowski, P. Kjaer, P.Sørensen, and J. Østergaard, "Variable speed wind turbines capability for temporary over-production," in Proc. IEEE PES General Meeting 2009, Calgary, Canada, pp. 1-7, Jul. 2009.

[12] M. Juelsgaard, J. Bendtsen, and R. Wisniewski, "Utilization of wind turbines for upregulation of power grids," IEEE Trans. on Ind. Electron., vol. 60, no. 7, pp. 2851-2863, Jul. 2013.

[13] A. D. Hansen, M. Altin, I. D. Margaris, F. Iov, and G. C. Tarnowski, "Analysis of the short-term overproduction capability of variable speed wind turbines," Renewable Energy, vol. 68, pp. 326-336, Mar. 2014.

[14] S. E. Itani, U. Annakkage, and G. Joos "Short-term frequency support utilizing inertial response of DFIG wind turbines," in Proc. 2011 IEEE Power \& Energy Society General Meeting, Detroit, USA, Jul. 2011.

[15] F. Hafiz and A. Abdennour, "Optimal use of kinetic energy for the inertial support from variable speed wind turbines," Renewable Energy, vol. 80, pp. 629643, Mar. 2015.

[16] IEEE Committee Report, "Dynamic models for steam and hydro turbines in power system studies," IEEE Trans. Power App. Syst., vol. PAS-92, no. 6, pp. 1904 1915, Nov. 1973.

[17] V. Ajjarapu, J. D. McCalley, D. Rover, Z. Wang, and $\mathrm{Z}$. Wu, "Novel sensorless generator control and grid fault ride-through strategies for variable-speed wind turbines and implementation on a new real-time simulation platform," Ph.D. dissertation, Dept. Elect. Eng., Iowa State Univ., Ames, Iowa, 2010.

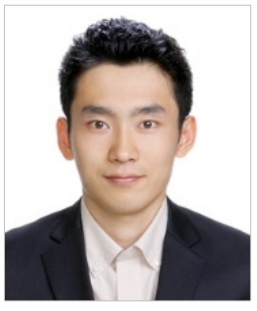

Dejian Yang $\mathrm{He}$ is currently pursuing a M.Sc. degree from the Department of Electrical Engineering at Chonbuk National University. $\mathrm{He}$ is also an assistant researcher at the Wind energy Grid-Adaptive Technology (WeGAT) Research Center supported by the Ministry of Science, ICT, and Future Planning (MSIP), Korea. His research interests include frequency support for wind power plants.

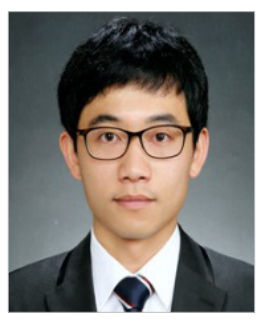

Jinsik Lee He received his B.S. and M.S. degrees from the Department of Electrical Engineering at Chonbuk National University, Korea, in 2011 and 2013. He is currently pursuing a Ph.D. degree at Chonbuk National University, and is also an assistant researcher at the WeGAT Research Center, supported by the MSIP, Korea. His research interests include plant-level control schemes for wind power plants.

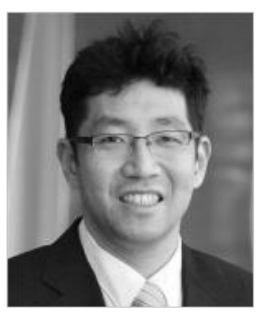

Kyeon Hur He received his B.S. and M.S. degrees in electrical engineering from Yonsei University, Seoul, Korea, in 1996 and 1998. He received his Ph.D. in electrical and computer engineering from The University of Texas at Austin in 2007. He was with Samsung Electronics as an R\&D engineer between 1998 and 2003, and was associated with the Electric Reliability Council of Texas (ERCOT) and Electric Power Research Institute (EPRI) between 2007 and 2010. He rejoined Yonsei University in 2010 and currently leads a research group for Smart Grid. His research interests include the integration of variable generation and controllable loads and flexible ac transmission systems.

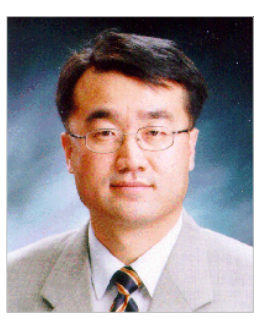

Yong Cheol Kang He received his B.Sc., M.Sc., and Ph.D. degrees in electrical engineering from Seoul National University, Korea, in 1991, 1993, and 1997, respectively. He has been with Chonbuk National University, Korea, since 1999 . He is currently a professor at Chonbuk National University and the director of the WeGAT Research Center supported by the MSIP, Korea. At present, he is a visiting scholar at the National Renewable Energy Laboratory in Golden, Colorado, and a member of the International Electrotechnical Commission working group TC88/WG27. His research interests include the development of control and protection techniques for wind power plants. 